\title{
Occurrence and Extent of Boscalid Resistance in Populations of Alternaria alternata from California Pistachio Orchards
}

\author{
Hervé F. Avenot ${ }^{\dagger}$ and Themis J. Michailides ${ }^{\dagger}$ \\ Department of Plant Pathology, University of California Davis, Kearney Agricultural Research \& Extension Center, Parlier, CA \\ 93648
}

Abstract

\begin{abstract}
Alternaria late blight (ALB) caused by Alternaria spp. is an annual disease problem in California pistachio and requires repeated applications of fungicides to prevent significant losses of pistachio foliage and nut quality. From 2003 onward, the succinate dehydrogenase inhibiting fungicide boscalid has played a key role in ALB management. The development of boscalid resistance in A. alternata populations was monitored from 2005 to 2012 in pistachio producing areas in California. A total of 1,765 singlespore isolates, collected from commercial and experimental pistachio orchards with or without a history of boscalid exposure, were tested in a radial growth assay in agar media amended with the discriminatory dose of $10 \mu \mathrm{g} / \mathrm{ml}$ of boscalid. The frequency of boscalid-resistant isolates in 2005 was $12 \%$ but increased significantly and remained stable toward the end of the survey period. Most of the resistant isolates exhibited a high level of resistance (R) to boscalid with percent of mycelial growth inhibition (PGI) values between 0 and 50\%, whereas significantly fewer isolates had an intermediate level (IR) of resistance $(50<$ PGI $<75 \%)$. The frequency of sensitive (S) isolates $(75<\mathrm{PGI}<100 \%)$ was generally the highest in orchards with no history of boscalid usage, whereas mean incidences of boscalid-resistant populations of Alternaria were 81, 92.4, 80.2 , and $98 \%$, in 2006, 2007, 2011, and 2012, respectively, in orchards that received a high number (at least three per season) of boscalid spray applications. In comparison, none to relatively low frequencies (0 to
\end{abstract}

$12 \%$ ) of resistance were observed in populations with no or limited exposure to Pristine, suggesting an air-movement of resistant spores through wind from treated to nontreated areas. In 2012, boscalid-resistant isolates were found practically in every sampled location in all counties, with the orchards in Fresno, Madera, Tulare, and King Counties being the locations with the highest frequencies of resistance (100\%). Monitoring of A. alternata AaSDHB, AaSDHC, and AaSDHD mutations in 286 boscalid-resistant phenotypes identified 11 mutations, leading to amino acid substitutions in AaSDHB (seven mutations: H277Y/R/L, P230A/ R, N235D/T), AaSDHC (one mutation: H134R), and AaSDHD (three mutations: D123E, H133R/P), with AaSDHB mutations being the most prevalent $(80 \%)$ ones throughout the survey period. The majority of isolates carrying these mutations exhibited the $\mathrm{R}$ phenotype toward boscalid. The increased prevalence of boscalid resistance in populations of A. alternata is a likely contributing factor to the inability of pistachio farmers to successfully control ALB with Pristine. Other factors implicated in the rapid and widespread occurrence of A. alternata boscalidresistant populations in California pistachios are further discussed.

Keywords: Alternaria late blight, chemical control, fungicide resistance monitoring, Pristine (boscalid + pyraclostrobin), succinate dehydrogenaseinhibiting fungicides (SDHIs), point mutations
In the United States, approximately $98 \%$ of commercial pistachio production occurs in the state of California. California growers have about 250,000 acres of pistachios in 22 counties, especially in the San Joaquin and Sacramento valleys, with the financial impact of the U.S. pistachio industry being about $\$ 1.7$ billion, according to the latest statistics (Perez and Minor 2018). For years, the California pistachio industry was based on a single female cultivar, Kerman, and a single male cultivar, Peters. Only in the last decade growers started planting Golden Hills and Lost Hills (female cultivars) and Randy (male cultivar), released by the University of California to the public. However, still the majority (approximately $>95 \%$ ) of plantings are Kerman with Peters male trees. As a result, the pistachio crop has a high level of genetic uniformity and is vulnerable to several diseases. In fact, several diseases caused by fungal pathogens are a major factor reducing the production and quality of this crop (Chao et al. 2001; Michailides and Morgan 1991; Michailides et al. 2016). One of these fungal diseases, namely, Alternaria late blight (ALB), is an

${ }^{\dagger}$ Corresponding authors: H. F. Avenot; hfavenot@ucdavis.edu and T. J. Michailides; tjmichailides@ucanr.edu

The author(s) declare no conflict of interest.

Accepted for publication 3 September 2019.

(C) 2020 The American Phytopathological Society annual production concern for commercial pistachio growers in California, and it is caused by three Alternaria species, namely, A. alternata, A. tenuissima, and A. arborescens (A. alternata complex) (Pryor and Michailides 2002). The pathogen infects both leaves and fruits, causes early defoliation, reduces yield, and stains nut shells (Chao et al. 2001). On leaves, the disease is characterized by the development of distinctly large necrotic lesions surrounded by chlorotic margins with a black center owing to the production of abundant fungal spores. Infection of leaves can occur in spring and early summer (latent infections), but symptoms usually develop in the late summer and fall. The disease can defoliate trees when the environmental conditions are optimal (Luo et al. 2007; Pryor and Michailides 2002). On fruit (pistachio), the disease starts as small necrotic spots in the lenticels. As the nuts develop, the lesions expand and cause the decay of the hulls. This results in severe staining of the nutshell and mold contamination of the kernel (Luo et al. 2007; Pryor and Michailides 2002). The major impacts for growers are weakening of the trees owing to premature defoliation and lowering of the quality of nuts by processors owing to dark shell staining. Losses owing to ALB can be significant in the central and southern California growing areas, where conditions in the orchards result in high humidity during the summer. The pistachio cultivar Kerman, which is planted in all commercial pistachio orchards in California, is susceptible to ALB. In the absence of resistant cultivars, cultural practices such as irrigation management and pruning to increase air movement and decrease air humidity in the orchard can help to manage ALB, but effective control of the disease has relied largely on multiple 
applications of respiratory-inhibiting fungicides (strobilurins or quinone-outside inhibitors [QoIs, FRAC number 11]) and succinate dehydrogenase inhibitors (SDHIs, FRAC number 7) during the growing season (Avenot et al. 2008a; Ma et al. 2003). Thanks to the discovery and release of the first QoI, azoxystrobin (a tradename in the United States is Abound) in 2000, high efficiencies in controlling ALB were obtained by pistachio growers, allowing them to increase the quality of nuts. However, this effective management with QoIs has become a challenge because the intensive use of Abound resulted in rapid selection and development of Alternaria azoxystrobin-resistant isolates. Resistance was owing to the presence of a G143A point mutation in the cytochrome bc1 that also conferred cross resistance to other registered QoIs (pyraclostrobin and trifloxystrobin). After a few years, resistance to QoIs in Alternaria populations became widespread across pistachio and almond growing areas in California, rendering these products ineffective and useless against ALB (Avenot et al. 2008a; Luo et al. 2007; Ma et al. 2003).

In 2003, the SDHI fungicide boscalid, in mixture with the QoI pyraclostrobin in the prepacked mixture Pristine (tradename in the United States), was introduced into ALB management programs as an anti-QoI-resistance strategy. This product became the only chemical alternative for pistachio growers at the time, and it provided a high efficiency in controlling ALB that primarily resulted from the activity of boscalid in inhibiting Alternaria QoI-resistant populations in pistachio orchards (Avenot et al. 2008a; Luo et al. 2007). Boscalid belongs to the SDHI fungicides, which specifically inhibit the complex II in the respiratory chain (Avenot and Michailides 2010; Kuhn 1984).

Because of the specificity of its mode of action and the ability shown by Alternaria species to adapt and develop resistance to single-site fungicides (Luo et al. 2007; Ma et al. 2003), there was a potential risk for boscalid resistance to develop in A. alternata in pistachio. The vulnerability of single-site fungicides to resistance development (Brent and Hollomon 1998) is well exemplified by the quick loss of QoI efficacy to resistance problems in ALB (Luo et al. 2007) and other fungal diseases, such as gray mold of grapevine (Leroux et al. 2010) or gummy stem blight of watermelon (Keinath 2012). This underlines the necessity to establish and develop a fungicide resistance monitoring program upon the market introduction and commercial use of a new chemical fungicide, which will increase its useful life (Brent and Hollomon 2000; Russel 2004). Such a monitoring program provides essential information for estimating and minimizing the risk of resistance development to chemical groups and for evaluating and implementing antiresistance management strategies (Russel 2004). Thus, a boscalid monitoring and management program was initiated by establishing and comparing Alternaria baseline sensitivities (Jutsum et al. 1998) determined prior to the commercial introduction of Pristine with sensitivity data of isolates collected from commercial pistachio orchards that have been treated with Pristine. These comparative studies led to the detection of field isolates of Alternaria with double resistance to boscalid and pyraclostrobin within two seasons after the first registration and commercial use of Pristine (Avenot and Michailides 2007; Avenot et al. 2008a). After the first detection and characterization of boscalid resistance, control failures following applications of Pristine continued to be reported in subsequent years in experimental and commercial pistachio orchards where spray programs were based on Pristine. These reports suggested that $A$. alternata isolates resistant to boscalid might be developing in these orchards, which could ultimately compromise the effectiveness of the boscalid-containing fungicide. Thus, monitoring of changes in boscalid sensitivity in A. alternata populations from pistachio-growing areas in California over time appeared crucial to understand the potential occurrence, evolution, distribution, and impact of resistance on ALB control with Pristine in pistachio orchards. These studies would allow us to anticipate and contend with resistance problems that could eventually result from the extensive use of Pristine against ALB.

In this present paper, we report on a study that aimed to monitor and determine the prevalence and molecular basis of boscalid resistance in populations of A. alternata collected between 2005 and 2012 across pistachio-growing areas in California. Factors implicated in the rapid and widespread occurrence of A. alternata boscalid-resistant populations in California pistachios are further discussed.

\section{Materials and Methods}

Collection of infected pistachio tissues and maintenance of A. alternata monosporic isolates. A. alternata isolates were isolated from pistachio tissues (leaves or buds) collected from major pistachio-growing regions during the pistachio growing seasons in 2005, 2006, 2007, 2008, 2010, 2011, and 2012 (Table 1, Fig. 1). Infected pistachio leaves or buds with ALB symptoms or latent infections were collected arbitrarily as disease distribution allowed. One isolate per one infected sample was recovered. A total of 1,765 single-spore A. alternata isolates were recovered from orchards with or without a history of Pristine (boscalid + pyraclostrobin) sprays. Spray records were not available from all these locations; generally, the experimental orchard at Kearney Agricultural Research \& Extension Center (KARE) (Parlier, CA; Fresno Co.) had been sprayed at least three times per year with Pristine starting in 2003 or 2004. In commercial orchards, Pristine had been applied at least three times per season according to initial label recommendations. These applications are usually made in June, July, and August, when conditions are very favorable for ALB development (Table 2).

Some of the isolates collected in 2008 and all from the 2012 collection were recovered from pistachio buds collected before the start of spraying for the corresponding seasons. In 2008, a total of 353 single-spore isolates of $A$. alternata were isolated from infected buds originating from 38 commercial pistachio orchards. In 2010, 134 single-spore isolates of $A$. alternata were collected from commercial pistachio orchards from different counties in California where Pristine had been used. In 2011, 111 isolates were collected from diseased leaves collected at the end of the growing season from the experimental pistachio orchard located at KARE. In 2012, 749 single-spore isolates of A. alternata were recovered from various pistachio orchards for a large-scale resistance monitoring survey. Among them, 173 were isolates from infected leaves collected at the end of the 2012 growing season from a commercial pistachio orchard located at KARE. This orchard received only one Pristine application that was made in 2012 in alternation with Luna Experience (fluopyram + tebuconazole) and Quash (metconazole). Two-hundred thirty isolates were collected from 31 commercial orchards located in several California counties (Table 1, Fig. 1). These isolates were recovered from plates used to perform the BUDMON technique (Michailides et al. 2014), which is primarily used to monitor Botryosphaeria spp. in pistachio orchards. The efficacies of registered and experimental SDHI-containing products were also evaluated during the last 2 years (independent sprays of Pristine, Merivon, Fontelis, and Luna products were applied in three to four sprays per season) at a grower orchard in Butte County. About 337 Alternaria isolates were recovered in 2012 from these plots using the BUDMON technique, by plating dormant pistachio buds. These plates were incubated at room temperature for 3 to 4 days.

To obtain single-spored isolates, plant tissues collected at the end of the growing season (leaves) or just before emerging at the beginning of April (buds) were sterilized for $3 \mathrm{~min}$ in a 10\% commercial bleach solution (Western Family Foods, Portland, OR). Tissues were then placed in $90-\mathrm{mm}$ Petri dishes containing acidified $(2.5 \mathrm{ml}$ of $25 \%$ [vol/vol] lactic acid per liter) Difco potato dextrose agar (APDA, $4.0 \mathrm{~g}$ of potato infusion, $20.0 \mathrm{~g}$ of dextrose, and $15 \mathrm{~g}$ of agar per liter), and plates were finally incubated at 22 to $24^{\circ} \mathrm{C}$ for 6 to 7 days. Different colonies were selected from Alternaria growing mycelium. To obtain spore suspension, a 5-mm mycelial plug was cut out with a cork borer, subsequently transferred into a 2-ml tube, and finally suspended in $2 \mathrm{ml}$ of sterile water. A. alternata singlespore isolates were obtained by spreading a $20-\mu l$ aliquot of the spore suspension on a water agar plate. After the plates were incubated at $22^{\circ} \mathrm{C}$ for $16 \mathrm{~h}$ in darkness, a small agar plug with a single germinating spore was picked up with a sterile surgical blade under a dissecting 
microscope $(15 \times)$ and transferred aseptically to a fresh APDA plate. Fungal colonies were then identified as A. alternata based on the morphological characteristics of the colony on PDA (Ma et al. 2003), and isolates were routinely stored on grade 40 silica gel or maintained on PDA plates (Davison Chemical, Baltimore, MD) at $4^{\circ} \mathrm{C}$.
Evaluation of A. alternata populations for sensitivity to boscalid. Sensitivity of the A. alternata populations to boscalid was assessed using an in vitro monitoring procedure by measuring the radial growth of each isolate on PDA amended with boscalid at a single discriminatory concentration of $10 \mu \mathrm{g} / \mathrm{ml}$, which clearly distinguishes

Table 1. Frequencies of boscalid-resistant isolates in Alternaria alternata populations collected from 2005 to 2012 from California pistachio orchards with or without a history of Pristine (boscalid + pyraclostrobin) usage

\begin{tabular}{|c|c|c|c|c|c|c|}
\hline \multicolumn{2}{|c|}{ Orchard } & \multirow[b]{2}{*}{ Year of isolation } & \multirow{2}{*}{$\begin{array}{c}\text { History of } \\
\text { Pristine sprays }\end{array}$} & \multirow[b]{2}{*}{ Number of isolates } & \multirow{2}{*}{$\begin{array}{c}\text { Frequency of } \\
\text { boscalid-resistant } \\
\text { isolates }(\%)\end{array}$} & \multirow[b]{2}{*}{ Phenotype $^{z}$} \\
\hline Name & County & & & & & \\
\hline Orc 4 & Fresno & 2005 & No & 49 & 2.04 & $1 \mathrm{R} ; 48 \mathrm{~S}$ \\
\hline Orc 1 & Kern & 2005 & Yes & 59 & 12.00 & $7 \mathrm{R} ; 52 \mathrm{~S}$ \\
\hline Orc 1 & Kern & 2006 & Yes & 42 & 81.00 & $34 \mathrm{R} ; 8 \mathrm{~S}$ \\
\hline Orc 2 & Kern & 2007 & Yes & 74 & 87.84 & $63 \mathrm{R} ; 2 \mathrm{IR} ; 8 \mathrm{~S}$ \\
\hline Orc 3 & Kern & 2007 & Yes & 25 & 96.15 & $23 \mathrm{R} ; 1 \mathrm{IR} ; 1 \mathrm{~S}$ \\
\hline Orc 1 & Tulare & 2007 & Yes & 27 & 89.00 & $21 \mathrm{R} ; 3 \mathrm{IR} ; 3 \mathrm{~S}$ \\
\hline Orc 2 & Tulare & 2007 & Yes & 53 & 94.34 & 45 R; 5 IR; 3 S \\
\hline Orc 3 & Tulare & 2007 & Yes & 88 & 96.59 & 80 R; 5 IR; 5 S \\
\hline JV & Madera & 2008 & No & 19 & 15.80 & $3 \mathrm{R} ; 16 \mathrm{~S}$ \\
\hline $\mathrm{T} 8$ & Tulare & 2008 & Yes & 106 & 75.50 & $80 \mathrm{R} ; 26 \mathrm{~S}$ \\
\hline A1 & Fresno & 2008 & Yes & 9 & 56.00 & $5 \mathrm{R} ; 4 \mathrm{~S}$ \\
\hline $\mathrm{F} 1$ & Kings & 2008 & Yes & 15 & 0.00 & $15 \mathrm{~S}$ \\
\hline Yolo & Yolo & 2008 & $\ldots$ & 30 & 0.00 & $30 \mathrm{~S}$ \\
\hline SQ1 & Kern & 2008 & No & 15 & 6.67 & $1 \mathrm{R} ; 14 \mathrm{~S}$ \\
\hline M1 & Madera & 2008 & Yes & 38 & 18.42 & $7 \mathrm{R} ; 31 \mathrm{~S}$ \\
\hline $\mathrm{C} 1$ & Madera & 2008 & Yes & 121 & 56.20 & $68 \mathrm{R} ; 53 \mathrm{~S}$ \\
\hline PAR & Fresno & 2010 & Yes & 28 & 60.7 & $9 \mathrm{R} ; 8 \mathrm{IR} ; 11 \mathrm{~S}$ \\
\hline TER & Tulare & 2010 & Yes & 12 & 25.00 & $2 \mathrm{R} ; 1 \mathrm{IR} ; 9 \mathrm{~S}$ \\
\hline COR & Kings & 2010 & Yes & 7 & 28.60 & $1 \mathrm{R} ; 1 \mathrm{IR} ; 5 \mathrm{~S}$ \\
\hline MAD & Madera & 2010 & Yes & 31 & 83.90 & $26 \mathrm{R} ; 5 \mathrm{~S}$ \\
\hline HAN & Kings & 2010 & Yes & 14 & 35.70 & $5 \mathrm{R} ; 9 \mathrm{~S}$ \\
\hline SHA & Kern & 2010 & Yes & 24 & 58.33 & $14 \mathrm{R} ; 8 \mathrm{~S}$ \\
\hline Unknown & - & 2010 & $\ldots$ & 18 & 88.90 & $16 \mathrm{R} ; 2 \mathrm{~S}$ \\
\hline $\mathrm{KAC} 1$ & Fresno & 2011 & Yes & 111 & 83.78 & 89 R; 4 IR; 15 S \\
\hline HFS & Butte & 2012 & Yes & 373 & 91.68 & $316 \mathrm{R} ; 26 \mathrm{IR} ; 30 \mathrm{~S}$ \\
\hline KAC2 & Fresno & 2012 & Yes & 173 & 85.70 & 99 R; 47 IR; 27 S \\
\hline OA2 & Fresno & 2012 & Yes & 18 & 100 & $16 \mathrm{R} ; 2$ IR \\
\hline $\mathrm{ME}$ & Fresno & 2012 & Yes & 7 & 100 & $6 \mathrm{R} ; 1 \mathrm{IR}$ \\
\hline TLB1 & Fresno & 2012 & Yes & 12 & 100 & $12 \mathrm{R}$ \\
\hline PB5 & Fresno & 2012 & Yes & 21 & 100 & $16 \mathrm{R} ; 5$ IR \\
\hline OA1 & Fresno & 2012 & Yes & 1 & 100 & $1 \mathrm{IR}$ \\
\hline $\mathrm{OHF}$ & Fresno & 2012 & Yes & 2 & 100 & $2 \mathrm{R}$ \\
\hline PB4 & Fresno & 2012 & Yes & 9 & 88.88 & $6 \mathrm{R} ; 2$ IR \\
\hline OR-62B & Fresno & 2012 & Yes & 1 & 100 & $1 \mathrm{R}$ \\
\hline NA & Kings & 2012 & Yes & 6 & 100 & $5 \mathrm{R} ; 1 \mathrm{IR}$ \\
\hline SVDW & Kings & 2012 & Yes & 5 & 100 & $4 \mathrm{R} ; 1 \mathrm{IR}$ \\
\hline AAB2 & Kings & 2012 & Yes & 14 & 100 & 12 R; 2 IR \\
\hline SF & Kings & 2012 & Yes & 3 & 67.00 & $2 \mathrm{IR} ; 1 \mathrm{~S}$ \\
\hline PJ & Madera & 2012 & Yes & 10 & 100 & $5 \mathrm{R} ; 5 \mathrm{IR}$ \\
\hline MB1 & Madera & 2012 & Yes & 14 & 93.00 & $6 \mathrm{R} ; 7 \mathrm{IR} ; 1 \mathrm{~S}$ \\
\hline C-101 & Tulare & 2012 & Yes & 22 & 100 & 16 R; 6 IR \\
\hline W-B & Tulare & 2012 & Yes & 12 & 100 & $11 \mathrm{R} ; 1 \mathrm{IR}$ \\
\hline S4B & Tulare & 2012 & Yes & 6 & 100 & $5 \mathrm{R} ; 1 \mathrm{IR}$ \\
\hline S4A & Tulare & 2012 & Yes & 2 & 100 & $2 \mathrm{R}$ \\
\hline S1A & Tulare & 2012 & Yes & 7 & 100 & $1 \mathrm{R} ; 6 \mathrm{IR}$ \\
\hline AW & Tulare & 2012 & Yes & 1 & 100 & $1 \mathrm{IR}$ \\
\hline BCC 105 & Tulare & 2012 & Yes & 20 & 95.00 & $9 \mathrm{R} ; 10 \mathrm{IR} ; 1 \mathrm{~S}$ \\
\hline BPR & Tulare & 2012 & Yes & 12 & 100 & $10 \mathrm{R} ; 2$ IR \\
\hline BR2 & Tulare & 2012 & Yes & 1 & 100 & $1 \mathrm{R}$ \\
\hline BTN & Tulare & 2012 & Yes & 3 & 100 & $3 \mathrm{R}$ \\
\hline $203 S$ & Tulare & 2012 & Yes & 8 & 100 & $8 \mathrm{R}$ \\
\hline $\mathrm{R} 2 \mathrm{C}$ & Unknown & 2012 & $\ldots$ & 6 & 100 & $6 \mathrm{IR}$ \\
\hline L4B & Unknown & 2012 & $\ldots$ & 7 & 100 & 7 IR \\
\hline L4A & Unknown & 2012 & $\ldots$ & 2 & 100 & 2 IR \\
\hline $\mathrm{C} 4$ & Unknown & 2012 & $\ldots$ & 4 & 100 & $4 \mathrm{IR}$ \\
\hline L1B & Unknown & 2012 & $\ldots$ & 2 & 100 & 2 IR \\
\hline L4D & Unknown & 2012 & $\ldots$ & 1 & 100 & $1 \mathrm{IR}$ \\
\hline
\end{tabular}


boscalid-resistant from boscalid-sensitive isolates (Avenot et al. 2008a, 2008b). Commercial formulation of boscalid (Endura; $70 \%$ active ingredient [a.i.]; BASF Corporation, Research Triangle Park, NC) was dissolved in sterile deionized water to provide a stock solution containing $10 \mathrm{mg}$ of a.i./ml and then stored at $4{ }^{\circ} \mathrm{C}$ until use. An aliquot of the stock solution was added to autoclaved PDA cooled to $50^{\circ} \mathrm{C}$ to produce the desired concentration of boscalid at $10 \mu \mathrm{g} / \mathrm{ml}$. Control plates were prepared by adding water to autoclaved and cooled PDA. Fungicide-amended and nonamended plates were then inoculated with a 5-mm mycelial plug taken from the edge of a 7-day-old culture of each isolate. Two replicate plates were used for each isolate, and the experiment was repeated twice. After incubation at $24^{\circ} \mathrm{C}$ in the dark for 7 days, colony diameter in each plate was measured in two perpendicular directions with the original mycelial plug diameter $(5 \mathrm{~mm})$ subtracted from each measurement. Mean percent inhibition of mycelial growth in 10$\mu \mathrm{g} / \mathrm{ml}$ boscalid-amended medium compared with the nonamended control medium was determined for each isolate. A. alternata isolates were classified into three categories based on their percentage of growth

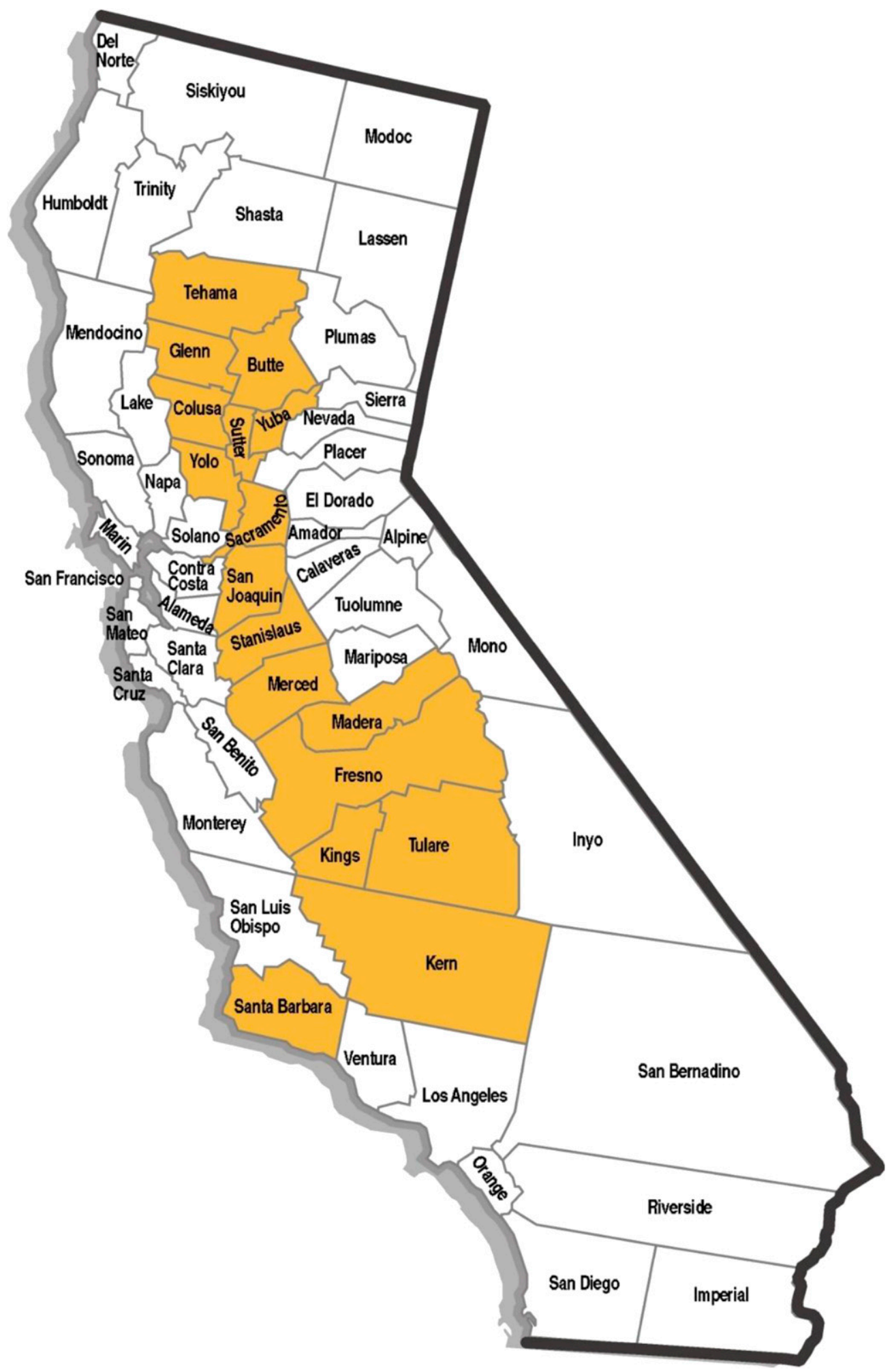

Fig. 1. Map showing locations of pistachio orchards in California counties. 
inhibition (PGI) relative to the control. Isolates with PGI values between 75 and 100 were classified as boscalid-sensitive (S); isolates with PGI values between 50 and 75 were considered to have an intermediate level of resistance (IR) to boscalid; and isolates with PGI values between 0 and 50 were considered highly resistant (R) to boscalid. Frequency of boscalid resistance was recorded for each orchard. Mean boscalid resistance incidence was calculated per each year of sampling and subjected to analysis of variance in SAS (version 9.3). Mean comparisons were conducted using the Fisher's protected least significant difference test.

Molecular diagnosis of boscalid-resistant phenotypes. To elucidate the molecular mechanism of resistance in boscalid-resistant isolates identified during the survey period, a total of 280 boscalidresistant phenotypes (Table 3) were screened for the presence of $S D H$ point mutations reportedly associated with boscalid resistance. Genomic DNA was extracted from mycelium of 10-day-old colonies of each A. alternata isolate using the FastDNA Kit (Qbiogene, Irvine, CA).

Polymerase chain reaction (PCR)-based assays were used to detect mutations in A. alternata SDHB (AaSDHB), SDHC (AaSDHC), and $S D H D(A a S D H D)$ genes of boscalid-resistant phenotypes detected in 2005, 2006, 2007, and 2010 (Table 3). Specifically, an allele-specific PCR developed by Avenot et al. (2008b) was used to test the presence of the point mutation ( $\mathrm{C}$ to $\mathrm{T}$ ) at nucleotide position 996 of the $A a S D H B$ gene, which results in an exchange of a histidine at position 277 by a tyrosine (H277Y). A cleaved amplified polymorphic sequence (CAPS) analysis with the restriction enzyme AciI (Avenot et al. 2008b) was used to detect the point mutation from A to $G$ at position 997 in the $A a S D H B$ gene that caused a change of the histidine residue at codon 277 to arginine (H277R) (Avenot et al. 2008b). A CAPS analysis with the restriction enzyme AciI (Avenot et al. 2009) was used to detect the point mutation (A to $G$ transition) at nucleotide position 490 in the $A a S D H C$ gene, which causes a replacement of a histidine residue at codon 134 by an arginine (H134R) (Avenot et al. 2009). CAPS analyses with the restriction enzymes XmnI and XcmI (Avenot et al. 2009) were used to detect the point mutations ( $\mathrm{C}$ to $\mathrm{A}$ at position 369 and $\mathrm{A}$ to $\mathrm{G}$ at position
398 ) in the $A a S D H D$ gene, which causes a substitution of an aspartate residue at codon 123 by a glutamic acid residue (D123E) and a substitution of a histidine residue at codon 133 by an arginine (H133R), respectively (Avenot et al. 2009). Portions of the $A a S D H B$, $A a S D H C$, and $A a S D H D$ genes potentially carrying these mutations were PCR amplified using specific primer sets as previously described (Avenot et al. 2008b, 2009). Respective PCR products were digested with specific restriction enzymes (New England BioLabs) according to the manufacturer's instructions. Digestion products were subsequently separated by electrophoresis on 3\% agarose gel in $1 \times$ TAE (40 mM Tris acetate, $1 \mathrm{mM}$ EDTA) buffer.

Sequencing of the $A a S D H B, A a S D H C$, and $A a S D H D$ genes was performed to identify mutations in boscalid-resistant phenotypes detected in 2011 (Table 3). Primer set 4 (5'-CGACGGACTCTAC GAATGC-3' / 5'-GCATGTCCTTGAGCAGTTGAG-3') (Avenot et al. 2008b) and two primer pairs (SdhC-SeqS2, 5'-CCATCTA CAAGCCCCAAATC-3' / SdhC-SeqR2, 5'-TCATCCGAGGAAGG TGTAGTAAAG-3'; and SdhD-SeqS1, 5'-CATCCAAGTCGTTCC GATATT-3' / SdhD-SeqR1, 5'-CGTTAGCCACGGTAACCACT$3^{\prime}$ ) (Avenot et al. 2009) were used to amplify and sequence regions of the $A a S D H B, A a S D H C$, and $A a S D H D$. PCR reactions were performed in a Mastercycler (Eppendorf) in 50- $\mu 1$ volumes containing $50 \mathrm{ng}$ of DNA, $50 \mathrm{mM} \mathrm{KCl}, 1.5 \mathrm{mM} \mathrm{MgCl} 2,10 \mathrm{mM}$ Tris- $\mathrm{HCl}$ (pH 9.0), $0.1 \%$ Triton X-100, dNTPs at $0.2 \mathrm{mM}$ each (Promega Corporation, Madison, WI), primers at $0.2 \mu \mathrm{M}$ each, and $1 \mathrm{U}$ of Pfu DNA polymerase (Agilent Technologies, La Jolla, CA). PCR products were separated by electrophoresis, purified, sequenced, and then analyzed, as previously described (Avenot et al. 2008b, 2009).

\section{Results}

Frequencies of boscalid-resistant isolates in A. alternata populations collected during the 2005 to 2012 pistachio growing seasons. An extensive monitoring of the presence and distribution of boscalidresistant isolates in A. alternata populations from California pistachioproducing regions (Fig. 1) was conducted from 2005 through 2012. Frequencies of boscalid-resistant isolates in commercially Pristinetreated and -untreated orchards were established for each county,

Table 2. Examples of Pristine (boscalid + pyraclostrobin) resistance management recommendations to control Alternaria in pistachio

\begin{tabular}{|c|c|c|c|c|c|}
\hline \multirow[b]{2}{*}{ Program } & \multicolumn{5}{|c|}{ Timing } \\
\hline & Dormant & April (bloom) & June & July & August \\
\hline Not recommended program & $\ldots$ & $\ldots$ & Pristine & Pristine, Pristine & Pristine \\
\hline Program option & None & None & None & $\begin{array}{l}\text { Pristine (full label rate only) }+ \\
\text { chlorothalonil }\end{array}$ & Switch + chlorothalonil ${ }^{\mathrm{z}}$ \\
\hline Program option & None & None & Pristine & Switch + chlorothalonil & $\begin{array}{l}\text { Pristine (full label rate only) }+ \\
\text { chlorothalonil }\end{array}$ \\
\hline Alternaria pressure & --- & --- & +++ & +++ & ++ \\
\hline
\end{tabular}

${ }^{\mathrm{z}}$ Switch $=$ cyprodinil (anilinopyrimidine) + fludioxonil (phenylpyrrole), and chlorothalonil (chloronitrile) is sold under the brand name Bravo.

Table 3. Sensitivity to boscalid of Alternaria alternata isolates collected from pistachio orchards with a history of Pristine use and associated amino acid residues in SDH target subunits

\begin{tabular}{lccc}
\hline Year of collection & Number of isolates & Phenotypes $^{\mathbf{y}}$ & ${\text { Genotypes: }{ }^{\mathbf{z}} \text { amino acid alterations in SDHB, SDHC, and SDHD proteins }}^{2005}$ \\
7 & $\mathrm{R}$ & 4 SDHB-H277Y; 3 SDHC-H134R \\
2006 & 38 & $\mathrm{R}$ & 9 SDHB-H277Y; 5 SDHB-H277R; 21 SDHC-H134R; 2 SDHD-D123E; 1 SDHD-H133R \\
2007 & 4 & $\mathrm{IR}$ & 2 SDHB-H277Y \\
& 137 & $\mathrm{R}$ & 123 SDHB-H277Y; 14 SDHB-H277Y; 10 SDHC-H134R \\
2010 & 1 & $\mathrm{IR}$ & 1 SDHB-H277Y \\
& 63 & & 46 SDHB-H277Y; 2 SDHB-H277Y; 16 SDHC-H134R; 1 SDHD-H133R \\
2011 & 6 & $\mathrm{IR}$ & 2 SDHB-N235D + SDHC-H134R; 1SDHC-H134R; 1 SDHB-N235D + SDHD-H133P \\
& 24 & $\mathrm{R}$ & 1 SDHB-H277L+N235D+P230R; 1 SDHB-N235D+P230A; 3 SDHC-H134R; 1 SDHB- \\
& & & N235T; 3 SDHB-N235D; 7 SDHB-N235D + SDHC-H134R; 1 SDHB-N235D + SDHD- \\
\end{tabular}

\footnotetext{
y Phenotypes were determined based on percent of mycelial growth inhibition (PGI) on boscalid-amended media: isolates with $75<\mathrm{PGI}<100 \%$ were considered sensitive (S) to boscalid; isolates with $50 \leq \mathrm{PGI}<75 \%$ were considered to have an intermediate level of resistance (IR); and isolates with $0 \leq \mathrm{PGI}<50 \%$ were considered highly resistant $(\mathrm{R})$.

${ }^{\mathrm{z}}$ Nucleotide mutations and corresponding amino acid alterations were detected using PCR assays or by sequencing of Alternaria alternata AaSDHB, AaSDHC, and AaSDHD subunits.
} 
and the results are presented in Table 1 . The annual frequency distributions of the response of Alternaria isolates to boscalid had a bimodal shape, and no departures from this were observed in any other years. Two discrete groupings of boscalid-sensitive (S; $75<$ PGI $<100 \%$ ) and boscalid-resistant $(0<$ PGI $<75 \%)$ phenotypes were identified. These resistant isolates could be further separated as highly resistant to boscalid (R; $0<$ PGI $<50 \%$ ) and intermediate level of resistance to boscalid (IR; $50<$ PGI $<75 \%$ ). The proportions of R and IR phenotypes for each sampled orchard during the period of sampling are shown in Table 1. In general, higher frequencies of boscalidresistant isolates were observed in Alternaria populations from orchards where Pristine had been used. There was an increase in the frequency of resistant isolates over the year, with the isolates exhibiting the $\mathrm{R}$ phenotype always predominating over the IR phenotype in all sampling years (Tables 1 and 4). The frequency of boscalid resistance was $12 \%$ in 2005 and increased very rapidly to $81 \%$ in 2006 within a single season in the same pistachio fields and sometimes over $90 \%$ in 2007 in some orchards (Tables 1 and 4). The frequency of resistant isolates increased significantly and remained stable toward the end of the survey period (Table 4). In contrast, the frequency of sensitive isolates was generally the highest in orchards with no history of boscalid usage, whereas boscalid-resistant populations of Alternaria were as high as $100 \%$ up to 2012 in orchards that received a high number of boscalid spray applications (Tables 1 and 4 ).

In 2007, for instance, frequency of resistance to boscalid varied from 26 to $88 \%$ in orchards in Kern and Tulare counties. Similar patterns were also observed in pistachio orchards the following years, in which a stepwise increase in the boscalid resistance frequency was observed (Tables 1 and 4).

In 2008, in order to have a better view of the distribution of resistant isolates in pistachio-growing areas, a total of 353 single-spore isolates of A. alternata were isolated from infected buds before any fungicides were applied during the season. Despite this, the frequencies of boscalid-resistant populations were still very high in some of these orchards (Table 1). Such frequencies, occurring early in the season before any application of fungicides, may have resulted from applications of Pristine in the previous season, which led to fewer boscalid-sensitive isolates and more boscalid-resistant isolates overwintering. It was interesting to note that only sensitive isolates were found in orchard F1, even though this orchard had been treated several times with Pristine. This may have reflected the spray program used at this site, which followed fungicide resistance management guidelines. In fact, a look at the fungicide sprays program used in this orchard in the previous 3 years (from 2005 to 2007) showed that Pristine applications were followed by an application of Scala SC, an anilinopyrimidine fungicide. Although the number of isolates tested was limited, this suggested that Scala SC was possibly efficient in controlling the boscalid-resistant populations if they were present. Resistance management strategy, in this case using another fungicide with a different mode of action in alternation with Pristine, may have accounted for the no shift observed, thus helping to reduce the occurrence or development of resistance in this particular orchard. All 30 Alternaria isolates collected in an orchard located in Yolo County were sensitive to boscalid (Table 1). Information on the fungicide use history from the Pest Control Advisor indicated that growers at this orchard have been using a lot of Abound each year for the past 5 years and typically using it at least three times in a season. In 2008, they used three applications of Abound and one Pristine application for the first time. This indicated that Pristine was a better material for managing QoI resistance and controlling ALB in this orchard.

Resistant isolates, probably brought up from a treated field, were detected at low proportions in some orchards with no history of boscalid exposure (Table 1), suggesting that these boscalidresistant isolates were probably brought up from Pristine-treated orchards by the wind.

Examination of the sensitivity profiles to boscalid of a 2012 collection of $230 \mathrm{~A}$. alternata isolates obtained from 31 commercial pistachio orchards where Pristine had been applied showed that over $90 \%$ of the isolates were resistant to boscalid (Tables 1 and 4). Among the 173 isolates collected from an experimental orchard at KARE with a history of fluopyram and Pristine use, $86 \%$ showed resistance to boscalid (Table 1), even though records showed that Pristine has not been used frequently in this orchard. Similarly, among the 373 isolates collected from a commercial orchard in Butte County that have been exposed to Pristine and other SDHIs (Fontelis, Merivon), 93\% were also resistant to boscalid (Table 1).

Occurrence of SDH mutations in A. alternata boscalidresistant phenotypes. PCR-based assays and sequencing analyses of the A. alternata SDHB, C, and D genes of 280 boscalid-resistant phenotypes identified a total of 11 mutations, leading to amino acid substitutions in AaSDHB (seven mutations: H277Y/R/L, P230A/R, N235D/T), AaSDHC (one mutation: H134R), and AaSDHD (three mutations: D123E, H133R/P), with SDHB mutations being the most prevalent $(80 \%)$ ones throughout the survey period. All the boscalidresistant phenotypes and associated $S D H$ mutations (genotypes), along with their distributions and proportions in the observed years, are presented in Table 3. Most of them were SDH mutants that exhibited the $\mathrm{R}$ phenotype to boscalid. Among the seven boscalid-resistant isolates detected in 2005, four and three displayed the AaSDHB-H277Y and AaSDHC-H134R genotypes, respectively. In 2006, AaSDHCH134R mutants were predominant (55\%). The genotype AaSDHB$\mathrm{H} 277 \mathrm{Y}$ was predominant in 2007 (90\%) and in 2010 (76\%) (Table 3 ). Noticeably, new mutations were found in some R and IR mutants identified in 2011, and some possessed a combination of mutations in the same or different AaSDH genes (Table 3).

\section{Discussion}

In order to anticipate and contend with boscalid resistance problems, a yearly survey was conducted to monitor the evolution and distribution of boscalid resistance in field populations of $A$. alternata from pistachio-producing areas in California. This fungicide, in mixture with pyraclostrobin in Pristine, helped to effectively manage ALB caused by the azoxystrobin-resistant G143A mutants (Karaoglanidis et al. 2011; Luo et al. 2007). Our monitoring data showed that the use of Pristine was quickly followed by the selection and development of boscalid-resistant isolates across pistachio orchards that were extensively treated with Pristine for many growing seasons. The incidence of boscalid resistance within the sampled Alternaria populations increased to high levels in individual pistachio orchards,

Table 4. Mean yearly incidence of boscalid resistance in populations of Alternaria alternata collected from California pistachio orchards

\begin{tabular}{|c|c|c|c|c|c|}
\hline \multicolumn{3}{|c|}{ Orchards } & \multicolumn{3}{|c|}{ Frequency $(\%)$ of boscalid resistance } \\
\hline History of Pristine use & Number & Year of collection & $\operatorname{Mean}^{\mathbf{z}}$ & Minimum & Maximum \\
\hline No (baseline) & 1 & 2005 & $2.0 \mathrm{c}$ & 2.0 & 2.0 \\
\hline \multirow[t]{7}{*}{ Yes } & 1 & 2005 & $12.0 \mathrm{c}$ & 12.0 & 12.0 \\
\hline & 1 & 2006 & $81.0 \mathrm{ab}$ & 81.0 & 81.0 \\
\hline & 5 & 2007 & $92.8 \mathrm{a}$ & 87.8 & 96.6 \\
\hline & 8 & 2008 & $28.6 \mathrm{c}$ & 0.0 & 75.0 \\
\hline & 7 & 2010 & $54.4 \mathrm{~b}$ & 25.0 & 88.9 \\
\hline & 1 & 2011 & $83.8 \mathrm{ab}$ & 83.8 & 83.8 \\
\hline & 33 & 2012 & $98.0 \mathrm{a}$ & 67.0 & 100.0 \\
\hline
\end{tabular}

\footnotetext{
${ }^{\mathrm{z}}$ Values within columns followed by the same letter are not significantly different $(P>0.05)$ according to Fisher's protected least significant difference.
} 
and this situation was detected in nearly all major counties of pistachio production.

The time taken for resistance to emerge and increase in frequency in a population after the introduction of a fungicide has been related to a number of factors, including the mode of action of the fungicide, elements of pathogen life cycle such as generation time and range of dispersal, the mode of use of the fungicide (e.g., dosage, mixtures and alternations, pleiotropic fitness costs associated with resistant genes, and the pathogen mutation rate toward resistance) (Dekker 1982; Gubbins and Gilligan 1999; Shaw 1992, 1993; Skylakakis 1985). Our survey showed that rapid shifts from boscalid-sensitive to boscalid-resistant isolates occurred in Alternaria populations, starting only 1 to 2 years after the commercial use of the boscalidcontaining product in pistachio. The high prevalence of boscalid resistance within populations of $A$. alternata was a likely contributing factor to the inability of pistachio farmers to control ALB with fungicide programs based on Pristine. In laboratory assays, boscalidresistant isolates could not be controlled with the labeled rate of Pristine, indicating that boscalid sensitivity shifts were adequate for the loss of control with field rate of the fungicide (Avenot et al. 2008a).

Several other factors likely contributed to the rapid selection and then development of boscalid resistance in A. alternata populations and resulting control failures of ALB with Pristine. These include the development of practical resistance of Alternaria to the QoIs, the exclusive reliance on Pristine, and the fitness of boscalid-resistant isolates. The second component of Pristine is the QoI pyraclostrobin, whose efficacy was already compromised by the time boscalid (Pristine) had been registered in pistachio, because Alternaria QoI resistance was already common across California pistachio-growing areas (Avenot et al. 2008a; Karaoglanidis et al. 2011; Luo et al. 2007). In most cases, as demonstrated previously (Avenot et al. 2008a) and confirmed in this study (data not shown), resistance to boscalid was found to be associated with resistance to pyraclostrobin. This double resistance to both classes of respiration inhibitors has resulted in difficulties in managing ALB disease with Pristine. Occurrence and development of boscalid-resistant isolates in fields where QoI resistance was already widespread have been reported in several fungal populations in various crops grown in the United States and in several other countries (Mallik et al. 2014; Veloukas et al. 2013).

The absence of registered fungicides from other chemical classes than QoI and SDHI to use in alternation with Pristine explained the overuse of this mixture. Therefore, the exclusive reliance on Pristine is another factor that likely caused the observed build-up of Alternaria populations resistant to boscalid in pistachio orchards. Environmental conditions are conducive for ALB development late in the season, and typically growers apply three to four applications per season. Initially manufacturer recommendations allowed for three applications of Pristine per season to manage ALB. Unfortunately, because Pristine was the only fungicide available for managing ALB at the time, pistachio growers had used it more frequently in consecutive years (three to four applications per season). Although exact application records were not available for all orchards, close examination showed that in locations having Alternaria populations with a high frequency of boscalid resistance $(>90 \%)$, Pristine had been used as a regular part of their fungicide programs for several years, since its registration in 2003, and in some cases in alternation with ineffective QoI fungicides. Not aware of potential resistance problems when ALB was seen developing at their orchards, many of Pristine sprays likely had resulted in incomplete disease control and, thus, maintained selection pressure on the pathogen population. Similar situations were observed in other fungal pathosystems, such as Alternaria solani in potato or Botrytis cinerea in strawberry, in which the incidence of boscalid resistance has been reported to increase in populations owing to exclusive and multiple applications of boscalid-containing product in a single season, which seriously affected its performance (Mallik et al. 2014; Veloukas et al. 2013).

Our results from this extensive monitoring of boscalid-sensitivity showed that the sampled A. alternata isolates were initially divided into two populations of boscalid-sensitive and -resistant isolates. Although the frequency of boscalid-resistant isolates was low at the beginning of the survey period, it quickly increased to high levels and remained relatively stable across California pistachio-growing areas. Regardless of the continuing development of Pristine resistance in populations of $A$. alternata, this product along with QoIs had continued utility for the control of two other major diseases of pistachio, namely Botryosphaeria panicle and shoot blight and Botrytis blossom and shoot blight. Therefore, populations of Alternaria were inadvertently and continually exposed to Pristine, which may have contributed to the stability of boscalid-resistant isolates in pistachios.

The partitioning of Alternaria isolates into two distinct groups of boscalid-sensitivity phenotypes is in accordance with the occurrence of a disruptive selection of resistance and indicated that resistance to boscalid was likely to be monogenic in nature, as expected for singlesite-inhibitor fungicides. This type of resistance is known to be one of the most important factors contributing to the rapid appearance and spread of fungicide-resistant alleles (Dekker 1982; Gubbins and Gilligan 1999; Milgroom 1990). Our monitoring of A. alternata AaSDHB, AaSDHC, and AaSDHD mutations in 286 boscalidresistant phenotypes detected throughout the sensitivity survey period confirmed that boscalid-resistant mutants with the $\mathrm{R}$ and IR phenotypes carried single or double point mutations in $A a S D H B$, $A a S D H C$, and/or $A a S D H D$ genes that encode the boscalid target proteins, as previously identified (Avenot et al. 2008b, 2009). Additional mutations at positions 230 and 235 in AaSDHB in particular were also revealed through sequencing. Most isolates exhibited the $\mathrm{R}$ phenotype toward boscalid, and along with IR isolates, they predominantly carried the AaSDHB-H277Y followed by the AaSDHC-H134R mutations. The occurrence of $A$. alternata field isolates displaying intermediate to high levels of resistance to boscalid, and carrying various mutations in the $S D H$ genes, as detected in this study, are consistent with several studies in Alternaria and other fungal species that showed the presence of several amino acid alterations (SDHB-H277Y/R/L; SDHCH134R; SDHC-S135R; SDHD-H133/R; and SDHD-D2123E) in SDHI target subunits, which were consistently found to be associated with various levels of boscalid resistance (Avenot and Michailides 2010; Avenot et al. 2008b, 2009, 2014; Lichtemberg et al. 2018; Pearce et al. 2019; Scalliet et al. 2012; Sierotzki et al. 2011; Yang et al. 2015)

Another factor that had impacted the rapid development of boscalid resistance is the abundant sporulation of these pathogens and the wind-blown dissemination of Alternaria spores (Avenot and Michailides 2007; Brent 1995). In fact, a high level of resistance was also detected in some pistachio orchards that had no Pristine spray application. This result suggested that boscalid-resistant isolates of the pathogen could be dispersed through wind. Even though some growers may have followed resistance management strategies, resistant isolates may have been introduced into their orchards from neighboring farmers who did not apply fungicides or used no resistance management practices. Therefore, it is critical that fungicide resistance management strategies be at local but also regional levels.

Another factor that had impacted the rapid development and persistence of boscalid resistance is the fitness of boscalid-resistant isolates. Previous studies with A. alternata indicated that boscalidsensitive and -resistant isolates (Avenot et al. 2008a) as well as QoI-sensitive and QoI-resistant isolates did not differ in their competitive fitness and that boscalid resistance remained stable following successive subculturing on fungicide-free PDA medium (Avenot and Michailides 2007; Avenot et al. 2008a; Karaoglanidis et al. 2011). Boscalid-resistant isolates were also recovered at high proportions from pistachio buds before any application of fungicides during the current season. This finding also suggested that resistant isolates may survive on infected pistachio buds, which provide initial inoculum for disease outbreaks in following seasons. These results associated with fitness implied that development of boscalid resistance after selection pressure by the fungicide would cause difficulty in controlling ALB.

This rapid development of boscalid resistance, as discovered in this survey, showed that the continued and exclusive reliance on 
Pristine has caused serious challenges for pistachio growers in effectively using this fungicide to manage ALB in locations with a resistant population. Therefore, there was a need for antiresistance strategies to be implemented to delay the appearance of resistant isolates in orchards where resistance had not developed or manage their increase in orchards where they had already emerged. Soon after the initial detection and emergence of boscalid resistance, extension efforts were made to increase growers' awareness about the status of boscalid resistance and of the necessity to carefully use Pristine in their ALB fungicide programs in order to prolong the effectiveness and usefulness of this fungicide. Thus, new fungicide management programs for areas with control failure were explored with the registration in 2004 of Switch (fludioxonil + cyprodinil), a prepacked mixture of the active ingredients cyprodinil (an anilinopyrimidine) and fludioxonil (a phenylpyrrole), and Scala (pyrimethanil) applied as a solo product (Table 2). In fungicide trials, Switch appeared effective in controlling ALB, and with the unique mode of action of its two components, it appeared as an excellent choice to include in antiresistance strategies to slow down, stop, or reduce the boscalid resistance build-up in Alternaria.

Based on the monitoring data and results of pistachio fungicide trials, boscalid-resistance management strategies were recommended to pistachio farmers to reduce the risk of boscalid-resistance development and extend the use of Pristine for ALB control in orchards where levels of boscalid resistance could still be manageable (Table 2). The recommendations included the need for growers to stop using Pristine in areas where resistance frequency exceeds $80 \%$; in other areas, growers should minimize the frequency of Pristine applications from three to four applications to one or two applications per season, use Pristine in rotation with fungicides with a different mode of action such as Switch, and tank mix Pristine with non-QoI products having a different mode of action than boscalid or with a broad spectrum fungicide (Table 2). Although Bravo (chlorothalonil) has been shown to be an effective partner to use in combination with Pristine, its phytotoxicity effect on pistachio leaves made it unsuitable for use against ALB (Michailides, personal communication). In this study, no resistant isolates were initially detected in an orchard where Pristine was alternated with the use of Switch or Scala, suggesting that the latter fungicides were possibly effective in controlling A. alternata boscalid-resistant isolates in this orchard. It is not known to what extent these products and recommendations were used or followed by pistachio growers, because monitoring of boscalid resistance in years following the registration and use of these products showed that frequencies of boscalid resistance continued to increase to high levels and resulted in the decline of Pristine efficacy. Recent monitoring of sensitivity of Alternaria isolates to fludioxonil and cyprodinil, both components of Switch, showed that these fungicides are still effective in controlling ALB (Avenot and Michailides 2015).

Boscalid was the first member of the SDHI group introduced against ALB in 2003. Since then, other new SDHIs were introduced, and the prevalence of boscalid-resistant populations in Alternaria in California pistachio orchards further complicates the efficient management of this disease using SDHI-related products (Avenot et al. 2014). This underlines the need to carefully use fungicides with a single-site mode of action in spray programs and focus on alternative management tactics for this disease in pistachio. In these areas, other fungicides from different cross-resistance groups such as demethylation inhibitors should be used (Avenot et al. 2016).

The information gathered from this work has shed light on the factors implicated in the occurrence, evolution, and widespread distribution of boscalid resistance in Alternaria populations in pistachioproducing regions in California. This has further shown the impact of resistance on fungicide performance in the field and helped to design and implement sound resistance-management programs for ALB control. Overall, the results presented here have shown Alternaria of pistachio as a model to study the problem posed by fungicide resistance and further revealed the challenges in predicting and in managing this phenomenon. The gained knowledge underlines the importance of monitoring programs and recognizing resistance issues as part of an integrated disease management strategy. It also showed evidence of the detrimental effect, caused by the lack of diversity or reliance on a single mode of action for disease control, to both growers and fungicide manufacturers and emphasized the need for new active ingredients.

\section{Acknowledgments}

We thank several pistachio farmers who allowed us to sample their orchards and Gary Weinberger (Pest Control Adviser), who provided some of the fungicide spray information for some of the orchards.

\section{Literature Cited}

Avenot, H., Morgan, D. P., and Michailides, T. J. 2008a. Resistance to pyraclostrobin, boscalid and multiple resistance to Pristine ${ }^{\circledR}$ (pyraclostrobin + boscalid) fungicide in Alternaria alternata causing Alternaria late blight of pistachios in California. Plant Pathol. 57:135-140.

Avenot, H., Sellam, A., and Michailides, T. J. 2009. Characterization of mutations in the membrane-anchored subunits AaSDHC and AaSDHD of succinate dehydrogenase from Alternaria alternata isolates conferring field resistance to the fungicide boscalid. Plant Pathol. 58:1134-1143.

Avenot, H., van den Biggelaar, H., Morgan, D., Moral, J., Joosten, M., and Michailides, T. J. 2014. Sensitivities of baseline isolates and boscalidresistant mutants of Alternaria alternata from pistachio to fluopyram, penthiopyrad, and fluxapyroxad. Plant Dis. 98:197-205.

Avenot, H. F., and Michailides, T. J. 2007. Resistance to boscalid fungicide in Alternaria alternata isolates from pistachio in California. Plant Dis. 91: 1345-1350.

Avenot, H. F., and Michailides, T. J. 2010. Progress in understanding molecular mechanisms and evolution of resistance to succinate dehydrogenase inhibiting (SDHI) fungicides in phytopathogenic fungi. Crop Prot. 29:643-651.

Avenot, H. F., and Michailides, T. J. 2015. Detection of isolates of Alternaria alternata with multiple-resistance to fludioxonil, cyprodinil, boscalid and pyraclostrobin in California pistachio orchards. Crop Prot. 78:214-221.

Avenot, H. F., Sellam, A., Karaoglanidis, G., and Michailides, T. J. 2008b. Characterization of mutations in the iron-sulphur subunit of succinate dehydrogenase correlating with boscalid resistance in Alternaria alternata from California pistachio. Phytopathology 98:736-742.

Avenot, H. F., Solorio, C., Morgan, D. P., and Michailides, T. J. 2016. Sensitivity and cross-resistance patterns to demethylation-inhibiting fungicides in California populations of Alternaria alternata pathogenic on pistachio. Crop Prot. 88:72-78.

Brent, K. J. 1995. Pages 1-48 in: Fungicide Resistance in Crop Pathogens: How Can It Be Managed? Fungicide Resistance Action Committee Monograph No.1. Groupement International des Associations Nationales de Fabricants de Produits Agrochimiques, Brussels, Belgium.

Brent, K. J., and Hollomon, D. W. 1998. Fungicide Resistance: The Assessment of Risk. Monograph No. 2. FRAC, Global Crop Protection Federation, Brussels, Belgium.

Brent, K. J., and Hollomon, D. W. 2000. Fungicide resistance management. Plant Dis. Res. 15:1-13.

Chao, C.-C. T., Parfitt, D. E., and Michailides, T. J. 2001. Alternaria late blight (Alternaria alternata) resistance in pistachio (Pistacia vera) and selection of resistant genotypes. J. Am. Soc. Hortic. Sci. 126:481-485.

Dekker, J. 1982. Can we estimate the fungicide-resistance hazard in the field from laboratory and greenhouse tests? Pages 128-138 in: Fungicide Resistance in Crop Protection. J. Dekker and S. Georgopoulos, eds. Pudoc, Wageningen, the Netherlands.

Gubbins, S., and Gilligan, C. A. 1999. Invasion thresholds for fungicide resistance: Deterministic and stochastic analyses. Proc. R. Soc. Lond. B Biol. Sci. 266: 2539-2549.

Jutsum, A. R., Heaney, S. P., Perrin, B. M., and Wege, P. J. 1998. Pesticide resistance: Assessment of risk and the development and implementation of effective management strategies. Pestic. Sci. 54:435-446.

Karaoglanidis, G. S., Luo, Y., and Michailides, T. J. 2011. Competitive ability and fitness of Alternaria alternata isolates resistant to QoI fungicides. Plant Dis. 95: 178-182.

Keinath, A. P. 2012. Differential sensitivity to boscalid in conidia and ascospores of Didymella bryoniae and frequency of boscalid-insensitive isolates in South Carolina. Plant Dis. 96:228-234.

Kuhn, P. J. 1984. Mode of action of carboximides. Symp. Ser. Br. Mycol. Soc. 9: 155-183.

Leroux, P., Gredt, M., Leroch, M., and Walker, A.-S. 2010. Exploring mechanisms of resistance to respiratory inhibitors in field strains of Botrytis cinerea, the causal agent of gray mold. Appl. Environ. Microbiol. 76:6615-6630.

Lichtemberg, P. S. F., Luo, Y., Doussoulin, H., and Michailides, T. J. 2018. Using allele-specific PCR for detecting multiple amino acid substitutions associated with SDHI resistance in Alternaria alternata causing Alternaria late blight in pistachio. Lett. Appl. Microbiol. 67:506-512.

Luo, Y., Reyes, H., Morgan, D. P., and Michailides, T. J. 2007. Using real-time PCR to survey frequency of the azoxystrobin-resistant allele G143A in Alternaria populations from almond and pistachio orchards in California. Pestic. Biochem. Physiol. 88:328-336. 
Ma, Z., Felts, D., and Michailides, T. J. 2003. Resistance to azoxystrobin in Alternaria isolates from pistachio in California. Pestic. Biochem. Physiol. 77: 66-74.

Mallik, I., Arabiat, S., Pasche, J., Bolton, M. D., Patel, J. S., and Gudmestad, N. C. 2014. Molecular characterization and detection of mutations associated with resistance to succinate dehydrogenase-inhibiting fungicides in Alternaria solani. Phytopathology 104:40-49.

Michailides, T., Morgan, D., and Doster, M. 2016. Foliar, fruit, and branch diseases. Pages 265-292 in: Pistachio Production Manual. L. Ferguson and D. Haviland, eds. University of California Agriculture and Natural Resources, Oakland, CA.

Michailides, T. J., and Morgan, D. P. 1991. An integrated approach for control of late blight caused by Alternaria alternata and management of mycoflora of pistachio through manipulation of irrigation practices. Pages 59-65 in: California Pistachio Industry Annual Report. Crop Year 1990-1991. California. Pistachio Commission, Fresno, CA.

Michailides, T. J., Morgan, D. P., and Puckett, R. D. 2014. Growers can reduce sprays during control of Botryosphaeria panicle and shoot blight of pistachio by using a leaf wetness model. Acta Hortic.: 179-184.

Milgroom, M. G. 1990. A stochastic model for the initial occurrence and development of fungicide resistance in plant pathogen populations. Phytopathology 80:410-416.

Pearce, T. L., Wilson, C. R., Gent, D. H., and Scott, J. B. 2019. Multiple mutations across the succinate dehydrogenase gene complex are associated with boscalid resistance in Didymella tanaceti in pyrethrum. PLoS One 14:e0218569.

Perez, A., and Minor, T. 2018. Fruit and Tree Nuts Outlook, FTS-367. U.S. Department of Agriculture, Economic Research Service, September 27, 2018.
Pryor, B., and Michailides, T. J. 2002. Morphological, pathogenic, and molecular characterization of Alternaria isolates associated with Alternaria late blight of pistachio. Phytopathology 92:406-416.

Russel, P. E. 2004. Sensitivity Baselines in Fungicide Resistance Research and Management. Monograph No. 3. FRAC, Crop Life International, Brussels, Belgium.

Scalliet, G., Bowler, J., Luksch, T., Kirchhofer-Allan, L., Steinhauer, D., Ward, K. Niklaus, M., Verras, A., Csukai, M., Daina, A., and Fonné-Pfister, R. 2012. Mutagenesis and functional studies with succinate dehydrogenase inhibitors in the wheat pathogen Mycosphaerella graminicola. PLoS One 7:e35429.

Shaw, M. W. 1992. How does spatial structure in populations affect the spread of fungicide resistance? Pages 136-147 in: Resistance 91: Achievements and Developments in Combating Pesticide Resistance. D. Hollomon, ed. Elsevier, London, U.K.

Shaw, M. W. 1993. Theoretical analysis of the effect of interacting activities on the rate of selection for combined resistance to fungicide mixtures. Crop Prot. 12:120-126.

Sierotzki, H., Frey, R., Morchoisne, M., Olaya, G., Mösch, M., and Scalliet, G. 2011. Sensitivity of fungal pathogens to SDHI fungicides. Pages 179-186 in: Modern Fungicides and Antifungal Compounds, Vol. VI. H. W. Dehne, H. B. Deising, U. Gisi, K. H. Kuck, P. E. Russell, and H. Lyr, eds. DPG, Braunschweig, Germany.

Skylakakis, G. 1985. Two different processes for the selection of fungicideresistant sub-populations. EPPO Bull. 15:519-525.

Veloukas, T., Markoglou, A., and Karaoglanidis, G. S. 2013. Differential effect of $S d h \mathrm{~B}$ gene mutations on the sensitivity to SDHI fungicides in Botrytis cinerea. Plant Dis. 97:118-122.

Yang, J. H., Brannen, P. M., and Schnabel, G. 2015. Resistance in Alternaria alternata to SDHI fungicides causes rare disease outbreak in peach orchards. Plant Dis. 99:65-70. 